\title{
Habitus, Kapital und Spielräume: Auf der Suche nach einem Oberarzt für die Irren- anstalt Hamburg-Friedrichsberg im Jahr 1897
}

\author{
Kai Sammet
}

\section{Summary}

In 1897 Hamburg was in search of an Oberarzt for the asylum Friedrichsberg who should function as the acting head of the head Wilhelm Reye (18331912). This search was part of the intended reformation of the outmoded psychiatric care in Hamburg. During this application procedure the Hamburg Physikus John Wahncau examined all possible candidates and applicants. The article explores the election process by using some sociological categories developed by Pierre Bourdieu (habitus, capital, field). The author argues that not only meritocratic attributes led to the choice of one candidate, but also his functional "fitting" into the field in Hamburg.

Keywords: Pierre Bourdieu; habitus; capital; field; German Psychiatry 19th century; asylum Hamburg-Friedrichsberg

\section{Zusammenfassung}

Im Jahr 1897 war Hamburg auf der Suche nach einem Direktorstellvertreter für die Irrenanstalt Friedrichsberg, um die als veraltet geltende psychiatrische Versorgung mit neuem Schwung zu versehen. Im Rahmen dieser Suche wurden seitens des Physikus John Wahncau eine ganze Reihe von möglichen Anwärtern und Bewerbern begutachtet. Der Aufsatz untersucht die Auswahl mit Hilfe einiger soziologischer Instrumente aus der Werkstatt Pierre Bourdieus (Habitus, Kapital, Feld). Dabei wird deutlich, dass nicht allein merito-

Dr. med. Kai Sammet, Universitätsklinikum Hamburg-Eppendorf, Martinistrasse 52, D-20246 Hamburg (sammet@uke.uni-hamburg.de). 
kratische Leistungsprinzipien, sondern die funktionale Passgenauigkeit für das Hamburger Feld bei der Auswahl des Bewerbers eine zentrale Rolle spielte.

\section{Einleitung: Gelegenheiten zu näherer Berührung}

«Darf ich mich Ihnen morgen nachmittag im Stadthaus vorstellen?»- Mit diesem Telegramm vom 21. Oktober 1897 versuchte Alexander Westphal (1863-1941) $)^{1}$, Sohn des berühmten Carl Westphal (1833-1890)2 , Assistent an der Charité und seit 1894 für Neurologie und Psychiatrie in Berlin habilitiert, direkten Kontakt zu Medizinalrat Johann Julius Reincke (1842-1906)3 , dem obersten Medizinalbeamten Hamburgs, herzustellen, um sich ihm sichtbar zu machen $^{4}$ : Esse est percipi. Kurz vorher war die andere Seite aktiv geworden. Im Mai 1897 war der Physikus John Wahncau (1862-1906) ${ }^{5}$ zur 22. Wanderversammlung der südwestdeutschen Neurologen und Psychiater in BadenBaden gereist. Dort bot sich ihm «reichlich Gelegenheit mit den jüngeren Theilnehmern und Privatdocenten in nähere Berührung zu kommen». Etwa 60 Kollegen waren anwesend, darunter auch einige «typische und tadellose Nerven $=$ und Badeärzte» ${ }^{6}$. Doch deretwegen war Wahncau nicht in den Kurort gekommen. Gesucht wurde ein Mann für die neue Stelle eines Oberarztes für die Hamburger Irrenanstalt Friedrichsberg, der nicht nur als Stellvertreter des Direktors Wilhelm Reye (1833-1912) ${ }^{7}$ fungieren, sondern als dessen Nachfolger aufgebaut werden sollte.

Die Auswahl eines Direktorstellvertreters an einer Irrenanstalt mag unwichtig erscheinen. Doch war ein solcher Posten mit Steuerungsmacht ausgestattet. Wenn Psychiatrie lokal verfertigt wird, dann stellen hochpositionierte Personen wichtige Schaltstellen dar. Die Besetzung einer Stelle meint den Auserwählten, insofern er diesen Posten besetzen kann. Dabei spielt dessen Biographie und Psychologie eine Rolle, doch diese Person ist «selbst in dem, was ganz persönlich an» ihr ist, «im wesentlichen eine Personifizierung der Anforderungen $»^{8}$, die zur Auswahl führen. So bietet sich eine

1 Cf. Kreuter 1996, III, 1562-1565.

2 Cf. Dierse 1995.

3 Cf. Pistor/Merkel 1906.

4 Telegramm Westphal an Reincke, 21.10.1897, Staatsarchiv Hamburg 352-3 Medizinalkollegium I H 3 c Begutachtung der Bewerbungen um Arztstellen bei den staatlichen Irrenanstalten 1858-1920, B1. 51 (nachfolgend: StHH Bewerbungen, Blattangabe).

$5 \mathrm{Zu}$ Wahncau siehe unten.

6 Reisebericht Wahncau, 24.5.1897, StHH Bewerbungen, B1. 36.

$7 \mathrm{Zu}$ Reye siehe unten.

8 Bourdieu/De Saint Martin 1978, 6f. Zitiert nach Krais/Gebauer 2002, 57. 
Kollektivbiographie an ${ }^{9}$. Die quantitative Untersuchung einer Gruppe durch Vergleich individueller Lebensläufe, die Darstellung typischer Merkmale, besonders sozialstatistischer Daten, erlaubt Aussagen über Übereinstimmungen und individuelle Besonderheiten, Offenheit oder Geschlossenheit, Rekrutierungsmodi des Kollektivs.

Aus zwei Gründen wurde dieser Zugang bei der Beschreibung dieser Stellenbesetzung nicht gewählt. Einerseits machen die Anzahl und die biographische Verfügbarkeit der «Spieler» (Bewerber, Empfehlende, Hamburger Entscheidungsträger) eine präzise Nachzeichnung jedes Lebenslaufs unmöglich ${ }^{10}$. Andererseits zielt mein Interesse in eine andere Richtung. Es geht weniger um objektiv fassbare Daten. Vielmehr verfolge ich zwei Ziele. Erstens soll an einer Fallstudie die Verwendung einiger soziologischer «Werkzeuge» probiert werden. Dabei geht es, zweitens, um einen Teilaspekt im Rahmen einer Untersuchung zur Hamburger Psychiatrie, die bisher wenig erforscht wurde ${ }^{11}$. Die Auswahl eines Bewerbers ist nicht nur eine Funktion seiner Sozialdaten, sondern eine Funktion ihrer Wahrnehmung und Einschätzung. Ein Bewerber 〈passt> oder 〈passt nicht〉, sein Habitus, seine Kapitalien, eine spezifische Situation entscheiden, wer in Frage kommt. Mittels einiger Begriffe, die von Pierre Bourdieu entwickelt wurden, lässt sich das Wechselspiel zwischen individuellem Bewerber, seiner Taxierung und der aktuellen lokalen Problemlage besser verstehen. Dabei geht es mir um die Verwendung, nicht um die Explikation oder Exegese dieser Begriffe ${ }^{12}$. Dennoch müssen sie zum besseren Verständnis skizziert werden ${ }^{13}$.

9 Das folgende nach Pieper 2003, $12 \mathrm{f}$.

10 Zur Quellenproblematik und zur Aufwendigkeit einer Kollektivbiographie: Pieper 2003, $15 \mathrm{f}$.

11 Eine umfassende Darstellung fehlt, Schmidt 1973 liefert eine Skizze; eine Ausnahme stellt die Zeit des Nationalsozialismus dar: vgl. zum Beispiel Rönn/Marien-Lunderup/Wunder/ Sonn/Otto/Billhardt/Dahmen 1993.

12 Mein Vorgehen findet Anhalt bei Bourdieu selbst. Seines Erachtens weicht die Verwendung von Begriffen von ihrer Explikation ab. Bourdieus Begriffe haben sich aus der Forschung entwickelt (siehe z.B. seine Ausführungen zum «kulturellen Kapital»: Bourdieu 1992, 53). Daher finden sich kontextabhängige Akzente. Begriffe sind «Werkzeuge» (Bourdieu/Chamboredon/Passeron 1991, 5), mit denen gearbeitet werden soll, sie dürfen nicht 〈fetischisiert〉 werden: Bourdieu 1993, 115. «Theorie» dürfe keine über der Empirie schwebende, einem Text oktroyierte Struktur sein. Sie müsse «überall und nirgends» sein: «in einer abschweifenden Anmerkung, im Kommentar eines alten Textes, in der Struktur des interpretierenden Diskurses selbst» (Bourdieu 1999, 284).

13 Ich lehne mich stark an Schwingel 2003, 59-102, an. Statt «Feld» benutze ich oft den Ausdruck «Spiel». So lässt sich die Dynamik des Ereignisses besser einfangen als mit dem andere Assoziationen weckenden Feldbegriff. 


\section{Habitus, Kapital, Spielräume}

Die Wahl eines Oberarztes für Friedrichsberg ist ein Spiel im Spiel, es geht um eine Postenverteilung im psychiatrischen Feld. In Bourdieus Soziologie fungieren Habitus und Feld als die «beiden Existenzweisen des Sozialen». Während der Habitus als «Leib gewordene» Geschichte soziale Fakten in die Individuen verlagert, sind Felder als Orte sozialer Praxis «Ding gewordene Geschichte» ${ }^{14}$. Soziale Praxis findet in spezifischen Feldern statt, «auto-

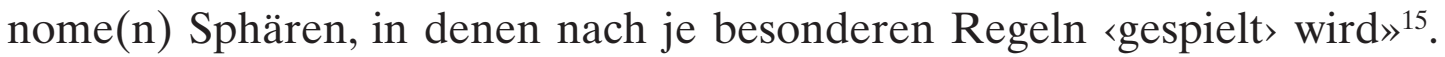
Jeder, der an einem Spiel teilnimmt, muss feldspezifische Regeln benutzen. So muss ein Unternehmer günstig produzieren, um Konkurrenten auszustechen, hier wird um Geld gespielt. In der Wissenschaft sticht das bessere Argument, hier wird um Wahrheit gespielt. Bei der Besetzung der Oberarztstelle in Hamburg geht es um (zukünftige) Macht- und Handlungsoptionen in einem lokalen Feld innerhalb der Anstaltspsychiatrie, aber auch um die Verwirklichung eines Biographieideals, Bewerber müssen signalisieren, dass sie der 〈Passende〉 sein könnten. Spieler in einem Feld sind nicht gleich. Zwei Positionierungen entscheiden über den Ort im Feld, die Profitchancen im Spiel.Erstens die Verfügung über Kapital,zweitens die relative Position jedes Spielteilnehmers zu allen anderen.

Mitentscheidend für eine Auswahl oder Kooptierung ist der Habitus eines Bewerbers. Die «habitus» werden von Bourdieu als «Systeme dauerhafter und übertragbarer Dispositionen», die in einer Lebensgeschichte strukturiert wurden und dann weiteres Verhalten strukturieren, konzipiert ${ }^{16}$. Der Habitus einer Person lässt sich als Integration dreier Aspekte beschreiben ${ }^{17}$.

Erstens gehen in ihn Wahrnehmungsschemata ein, sie strukturieren die Alltagswahrnehmung (so sieht man z.B., zu welcher Klasse jemand gehört). Zweitens gehören zum Habitus Denkschemata, die (1.) Alltagstheorien und Klassifikationsmuster der sozialen Umgebung, (2.) ethische Muster («Distanzlosigkeit» z.B. ist verwerflich, signalisiert fehlende Erziehung) und (3.) ästhetisch-geschmackliche Muster ${ }^{18}$ umfassen. Drittens folgen Handlungsschemata, die die Erzeugung von Handlungen, das Verhalten, strukturieren. In der nachfolgenden Skizze lassen sich diese in der Praxis engverknüpften Schemata aufgrund der Quellenlage nur fragmentarisch

14 Bourdieu 1985, 69.

15 Schwingel 2003, 83.

16 Bourdieu 1987, 98.

17 Zum folgenden siehe besonders Schwingel 2003, 62-65. Zur differenzierten Darstellung vgl. Krais/Gebauer 2002.

18 Es ist offensichtlich, dass sich beide Kategorisierungen (ethisch, ästhetisch) oft kaum trennen lassen. Ästhetische Urteile werden ethisch aufgeladen, um sie gegen Kritik zu immunisieren. 
erkennen. Wahrnehmungs- und Denkschemata der Bewerber werden nur in Ansätzen sichtbar. Auf der Seite der Hamburger Medizinalbehörden lassen sich diese besser rekonstruieren: Der Habitus einer Person wird wahrgenommen, er wird erspürt, man erkennt intuitiv, welches Verhalten dem in den Blick genommenen Anwärter «versperrt» (oder möglich) ist ${ }^{19}$. Ein Oberarztaspirant muss in einen spezifischen Rahmen und Kontext passen, er passt, wenn sein «Erscheinungsbild ${ }^{20}$ passt. Dabei geht es um ein komplexes Gesamtbild, nicht um eine einzelne Eigenschaft. Die vorzeigbare Leistung, jenes an der spezifischen Laufbahnposition, die der Bewerber einnimmt, ablesbare institutionalisierte kulturelle Kapital spielt eine wichtige, aber nicht entscheidende Rolle ${ }^{21}$. Es geht um eine Person, die in ihrer Position als Anwärterin, in Altersprofil (er darf nicht zu jung sein, sonst erschiene er als zu unerfahren, aber auch nicht zu alt, sonst wäre er zuwenig biegsam), Ausbildung und wahrgenommenem Verhalten am wahrscheinlichsten in der Hamburger Zukunft funktioniert. Der Habitus ist bis in Gesten, Bewegung verkörpert, entscheidend für eine Kooptierung können Dress- und Benimmcodes $^{22}$, Umgangsformen, Kenntnis und Beachtung ungeschriebener Regeln, Allgemeinbildung, Souveränität des Auftretens $\operatorname{sein}^{23}$. Gewählt wird ein Bewerber nach Ähnlichkeit. Das ist funktional, mit jemandem, der einem Hamburger Bürger ähnelt, lassen sich Probleme leichter lösen. Jemanden auszuwählen meint Vertrauensvorschuss. Das Problem der Wahl ist ein Problem des Vertrauens. Vertrauen dient der Komplexitätsreduktion, ist es vorhanden, so gibt es mehr Handlungsmöglichkeiten, dies erhöht die Komplexität des sozialen Systems, mithin «die Zahl der Möglichkeiten, die es mit seiner Struktur vereinbaren kann», aber Vertrauen zu erweisen bedeutet, Zukunft vorwegzunehmen. Doch genau das ist unmöglich, denn man erwählt unter Umständen einen Fremden zum Oberarzt, von dessen Funktionieren keine Kenntnis erhältlich ist. Vertrauen ist somit «riskante Vorleistung». Da Zukunft offen ist, wird das «Vergegenwärtigungspotential der Menschen (überfordert)»: Man weiss nie, welche Fehler jemand zukünftig produziert. Hier hilft nur abgesichertes Vertrauen. Blind darf es nicht sein, die eigene Risikofreude muss gezügelt sein. Das geschieht dadurch, dass man «sich sein Objekt mit Hilfe von Symbolen der Vertrauenswürdigkeit nahebringt». Ein wahrgenommener Habitus ist ein Symbolbündel der Vertrauens- oder Misstrauenswürdigkeit. Wenn Vertrauen «überzogene Information» ist, darauf

19 Cf. Bourdieu 1989, $26 f$.

20 Schwingel 2003, 60.

21 Hartmann 2001, 192. - Zum Kapitalbegriff Bourdieus siehe unten.

22 Vergleiche Hartmann 2002, insbesondere 371-376.

23 Hartmann 2001, 184. 
beruht, «dass der Vertrauende sich in gewissen Grundzügen schon auskennt, schon informiert ist, wenn auch nicht dicht genug, nicht vollständig, nicht zuverlässig», dann braucht Vertrauen die «Vertrautheit der Vertrauensperson» zur Basis, Vertrautem vertraut man eher als Fremdem. Dabei unterstellt man, dass der andere sein unendliches Handlungspotential gemäss seiner Persönlichkeit einsetzt, also: «im Sinne der Persönlichkeit, die er als die seine dargestellt und sozial sichtbar gemacht hat. Vertrauenswürdig ist, wer bei dem bleibt, was er» über sich mitgeteilt hat - ähnliche «habitus» erkennen einander, das Erscheinen als Person setzt ein Mindestmass an Vertrauen voraus, «nämlich das Vertrauen, nicht fehlgedeutet zu werden, sondern im grossen und ganzen so angenommen zu werden, wie man sich zu zeigen wünscht» ${ }^{24}$. Westphals Wunsch, sich Reincke zu zeigen, wird hier verständlich ebenso wie die Ressource persönlicher Bekanntheit und Empfehlung ${ }^{25}$. Doch persönliches Erscheinen im Stadthaus allein genügt nicht, um Oberarzt zu werden. Man muss mehr ins Spiel bringen.

Bourdieu unterscheidet im wesentlichen vier Kapitalarten. Zwar fasst er Kapital als «akkumulierte Arbeit» auf, doch ist sein Kapitalbegriff nicht ökonomistisch verkürzt, vielmehr wird in ihm beschrieben, welche Handlungs-, das heisst Profitchancen jeweils ein Spieler in einem Feld hat ${ }^{26}$. Ökonomisches Kapital, wie zum Beispiel Geld und Besitz, ist «unmittelbar und direkt in Geld konvertierbar ${ }^{27}$. Die zweite Kapitalart, kulturelles Kapital, existiert in drei Formen. Erstens findet es sich in objektiviertem Zustand, zum Beispiel in Maschinen. Zweitens ist es in inkorporiertem Zustand existent, hier handelt es sich um dauerhafte Dispositionen des Organismus, erworben in einem Sozialisations- und Bildungsprozess und so zu einem Teil des eigenen Habitus geworden. Diese Kulturakkumulation ist personengebunden, investiert wird zum Erwerb vor allem Zeit, aber auch Geld, denn man muss von ökonomisch notwendiger Arbeit freigesetzt sein. Der Erwerb kulturellen Kapitals beginnt in der Primärfamilie, schon hier kann positiv «als gewonnene Zeit und Vorsprung» ein hübsches Sümmchen angehäuft sein, das später in der Konkurrenz von Mitbewerbern um Stellen als der Person als Fähigkeit oder Talent anhaftendes Verdienst gerechnet und genutzt wird. Schliesslich, drittens, taucht kulturelles Kapital in institutionalisiertem Zustand auf. Hiermit sind institutionell, also gesellschaftlich und staatlich legitimierte Inkorporationen kulturellen Kapitals wie Schul-, Hochschulabschlüsse oder absolvierte Laufbahnpositionen gemeint.

24 Luhmann 1973, 8, 12, 21, 31, 34, 40f.

25 Siehe dazu weiter unten.

26 Bourdieu 1985, 10.

27 Bourdieu 1992, 52. 
Spezifische Formen inkorporierten und institutionalisierten kulturellen Kapitals stellten die Zugangsvoraussetzungen für eine Bewerbung um den Hamburger Stellvertreterposten dar. Grundsätzlich war das von den Bewerbern ins Spiel zu bringende Kapital ähnlich hoch, denn an diesem Spiel konnten nur Mitglieder einer homogenen gesellschaftlichen Gruppe teilnehmen: akademisch ausgebildete Ärzte im Wilhelminischen Kaiserreich,Angehörige des Bildungsbürgertums, einer Sozialformation, zu der circa 1\% der deutschen Bevölkerung zu zählen war, humanistisch gebildet, vielfach privilegiert, zumeist staatsnah - eine kleine, kulturell prägende Gruppe. Wenn sich dort auch relativ viele Aufsteiger aus mittleren Bürger- oder gar Kleinbürgerschichten fanden, so war die Selbstrekrutierungsquote der Ärzte aufgrund der langen und teuren Ausbildung hoch, zwei Drittel stammten aus den oberen Mittelklassen, nicht selten aus Arztfamilien, so dass hier «relative Exklusivität vorherrschte» ${ }^{28}$. Die seit 1880 ansteigenden Studentenzahlen, auf die mit einer Verschärfung der Anforderungen zum Erhalt der Exklusivität reagiert wurde, verstärkten die Tendenz zur Bereitstellung eines homogenen Habitus am Markt ${ }^{29}$.

Inkorporiertes und institutionalisiertes kulturelles Kapital kann via Laufbahn in ökonomisches Kapital konvertiert werden. Doch war nicht sicher, dass sich die zeitraubende Investition in Bildung amortisierte, auch wenn Aufstiegsprekarität durch lebenslaufspezifisch typische Positionen abgesichert werden sollte: zuerst Volontär, dann Assistent, dann Oberarzt/zweiter Arzt, dann Direktor ${ }^{30}$. Wenn nun Bewerber ähnlich viel institutionalisiertes Kapital ins Spiel bringen, so müssen Ungleichheiten wahrgenommen, mussten aus «kontinuierlichen Verteilungen [...] diskontinuierliche Gegensätze» konstruiert werden, bei denen die «Unterschiede aus der physischen Ordnung der Dinge» (z.B. Gleichheit des Bildungsabschlusses) in die «symbolische Ordnung signifikanter Unterscheidungen ${ }^{31}$ kippen. Das zeigt sich an der Bewertung der Anwärter. An der genuin agonalen Konkurrenzsituation einer Stellenausschreibung lässt sich ein Habitusaspekt illustrieren, der ansonsten erschlossen werden muss. Die in ihn eingegossenen Wahrnehmungs-, Denk-, Handlungsschemata bleiben implizit, sie bleiben Wahrnehmendem wie Wahrgenommenem als in ihren Körper und in ihr Verhalten

28 Wehler 1995, 730-735, Zitat: 734.

29 Siehe zur Lage und Entwicklung des Ärztestandes im Kaiserreich: Herold-Schmidt 1997.

30 Dieses Viererschema ist grob. Es gab erhebliche regionale Unterschiede, die in unterschiedlichen Bezeichnungen, damit unterschiedlichen Stellenbeschreibungen mit unterschiedlichen Besoldungs-, Anstellungsmustern resultierten. Hier geht es um das Muster, das Nähe oder Ferne zum höchsten Posten der Stufenfolge definiert. Die Hamburger Stelle ist das Sprungbrett zum Direktor, die Bewerber sind «Anwärter».

31 Bourdieu 1982, 284. 
absedimentierte soziale Formung verborgen. In einer Bewertungssituation werden sie, wenn auch fragmentarisch, deutlich.

Wenn Bewerber ähnliche Voraussetzungen für einen Posten mitbringen, dann konstruiert der Bewertende Unterschiede über eine oft entscheidende Kapitalart. Soziales Kapital ${ }^{32}$ existiert als dauerhaftes Netz von «Beziehungen gegenseitigen Kennens und Anerkennens». Man gehört zur Gruppe der Psychiater, zu den in der prekären Mittellage sich befindenden Jungen, die auf dem Sprung sind. Um hier Distinktionsgewinne zu erzielen, um einen Aufstiegsposten konkurrieren zu können, sind Verbindungen, Netzwerke unabdingbar. Der Umfang dieses Kapitals hängt von der Ausdehnung des Netzes und vom «Umfang des (ökonomischen, kulturellen oder symbolischen) Kapitals, das diejenigen besitzen», mit denen man in Beziehung steht, ab. Soziales Kapital ist flüchtig, seine Reproduktion erfordert Beziehungsarbeit durch Austauschakte zur Bestätigung gegenseitiger Anerkennung. Soziales Kapital übt einen Multiplikatoreffekt auf andere Kapitalarten aus. Netzpflege erleichtert den Zugang zu einer guten Universität, das erhöht die Eintrittswahrscheinlichkeit in einen höheren Posten. Soziales Kapital ist ebenfalls nur prekär in Geld konvertierbar. Der Wunsch nach Konvertierbarkeit wie das Wissen um die Flüchtigkeit sozialen Kapitals gehen in das Verhalten der Anwärter ein - das zeigt Westphals Verhalten. Der Kontakt zu Reincke im Moment der Auswahl sollte ein persönliches Kennen ermöglichen, davon versprach sich Westphal Anerkennung, die dann, über eine Empfehlung Reinckes an den richtigen Stellen, in einen Posten umsetzbar sein sollte.

Aus der Ordnung der physischen Dinge in die Ordnung des Symbolischen gerät man weiter, wenn man eine weitere, vom sozialen Kapital nur unscharf zu trennende Kapitalart, symbolisches Kapital oder kurz: Prestige oder Renommee ${ }^{33}$, ergänzt. Hier findet die Benutzung von Empfehlungen durch Anwärter ihren Platz. Blosses Kennen genügt nicht. Man muss auch anerkannt, wertgeschätzt werden. Über einen hochkarätigen Empfehlenden soll eine hohe Quantität symbolischen Kapitals angezeigt werden. Die Empfehlung des allseits anerkannten Bürgen, zum Beispiel eines älteren, auf der Karriereleiter schon oben angekommenen Kollegen, weist Kapital zu, das in einer Bewerbungssituation umgemünzt werden soll.

Aus Sicht der Bewerber selbst stand viel auf dem Spiel. Instruktiv sind hier Bemerkungen in Alfred Hoches (1865-1943) Autobiographie ${ }^{34}$. Sein bürgerlicher Hintergrund war eine gute Voraussetzung, dennoch war seine Lage in

32 Zum folgenden: Bourdieu 1992, 63-70, folgende Zitate: 64, 67.

33 Bourdieu 1985, 11.

34 Zum folgenden: Hoche 1941,119-142, Zitate: 140, 142, 112f. - Zu Hoche siehe unten. 
seiner Selbstwahrnehmung prekär. 1865 als Sohn eines Pfarrers geboren, wurde er nach Assistententätigkeit in Gynäkologie und Pädiatrie, die ihm durch den Tod der jeweiligen Chefs nicht das erhoffte door opening ermöglichte, 1890 in Heidelberg Assistent bei Carl Fürstner (1848-1906) ${ }^{35}$. Er ging mit diesem nach Strassburg, habilitierte sich, wurde Oberarzt, doch überwarf er sich mit seinem Chef: Das Verhältnis zu Fürstner trübte sich «in der für älter werdende Assistenten fast gesetzmässigen Weise», Fürstner wurde zunehmend «mit meiner wachsenden inneren Selbständigkeit eifersüchtig». Hoche liess sich als Nervenarzt nieder, betrachtete sich als «Gestrandeten», stand vergeblich auf Berufungslisten. Hoche beschrieb hier die «Anwärterschaft» im universitären oder Chefarztfeld: Auch in anderen Berufen gebe es Wartezeiten und Enttäuschungen, doch nur hier gehe es darum, «ob man im Chausséegraben liegen bleibt oder sein Lebensziel erreicht». Leistungs-, Aufstiegsprinzipien waren tief im wilhelminisch-bürgerlichen Habitus verankert. Hoche adelte «das Streben, eine Stufe höher zu kommen», zu einem «inneren» Gesetz des «natürlichen Menschen, wie er nun einmal ist», der Aufstieg hatte wohlgeordnet nach der «welten-uralte[n] Abstufung von Lehrling, Geselle und Meister» abzulaufen. Es ging nicht nur um eine für die Ehre wichtige Stufe, nicht nur um Autonomie, sondern auch um die Realisierung eines Biographie- und Familienideals (wie auch um männliche Sexualität in geregelten Bahnen). Eine Heirat als Assistent war unmöglich, erst eine Festanstellung erlaubte Familiengründung ${ }^{36}$. Viel stand auf dem Spiel im Spiel, bei den Anwärtern wie in Hamburg. Erst die dortige spezifische Situation macht die späteren Bewertungen der Anwärter plausibel.

\section{Konturen des Hamburger Felds, Spielregeln im Hamburger Spiel}

Die verheerende Choleraepidemie des Sommers 1892 hatte den desolaten Zustand der Gesundheitshygiene, des Medizinalwesens und massive soziopolitische Verwerfungen einer handelskapitalistischen Grossstadt gezeigt ${ }^{37}$. Sicher war die Cholera nicht der Motor gesellschaftlicher Reformen. Bedeutsamer war die durch den Hafenarbeiterstreik 1896/97 ausgelöste Beunruhi-

35 Zu Fürstner: Kreuter 1996, I, 419-421.

36 Dieses Heiratsproblem könnte ein Grund gewesen sein, warum Hoche sich mit Fürstner überwarf: Kirstein 1997, 38f. Die Bedeutung des Verlassens des finanziell wie karrierespezifisch misslichen Assistentenstatus wird auch in der Autobiographie Robert Wollenbergs (der schliesslich die Hamburger Stelle erhielt, siehe unten) deutlich. Die ersten Sätze über seine Berufung nach Hamburg handeln nicht von seiner Stelle, sondern beschreiben seine Erleichterung, dass er nun seine Braut heiraten konnte: «Wir waren überglücklich. Denn nun war unsere Vereinigung in greifbare Nähe gerückt [...]» (Wollenberg 1931, 90).

37 Cf. Evans 1990, insbesondere 639-676. 
gung in Hamburgs Bürgertum. Einige Veränderungen gehörten in einen längeren, bis in die 1880er Jahre reichenden Transformationsprozess des Stadtstaates als Teil des neuen Kaiserreichs. Reformen im Medizinalwesen waren schon lange geplant, doch immer wieder auf die lange Bank geschoben worden, so zum Beispiel die Neufassung der alten, seit 1818 geltenden Medizinalordnung. Vorarbeiten zu einer neuen gingen bis in die 1840er Jahre zurück. Doch erst in den 1890er Jahren ging man mit Elan an die Sache, 1900 trat eine neue Medizinalordnung in $\mathrm{Kraft}^{38}$. Hier hatte die Choleraepidemie eine Katalysatorfunktion. Reincke, der das Medizinalwesen modernisieren wollte, löste den alten Medizinalrat ab, der mitverantwortlich für das desolate Krisenmanagement im Sommer gewesen war. Zunehmende Verrechtlichung durch erforderliche Rechtsanpassungen an das Reich und erhöhte Kontrolle des Medizinalwesens in Hamburg, das bis dato dem Laisser-faire huldigte und möglichst wenig regeln wollte, waren die wichtigsten Kennzeichen dieser Reformen.

Doch stellten sich der Psychiatrie Hamburgs noch gesonderte Probleme. Irrenanstalten gerieten um 1890 in toto unter Druck. Vermeintlich oder tatsächlich unrechtmässige Einweisungen und Behandlung in als düstere Kerker verschrieenen Asylen wurden einer von Laien initiierten antipsychiatrischen Kritik unterzogen. Hier wurden Modernisierungsängste in fassbare Formen übersetzt, in denen sie ihren Weg in Tagespresse, Broschüren und parlamentarische Debatten fanden ${ }^{39}$. Reincke versuchte den Modernisierungsbedarf, den die Choleraepidemie gezeigt hatte, und die Kritik einer aufmüpfigen Öffentlichkeit in eine Reform lokaler Fürsorge umzusetzen. Bestärkt wurde er darin durch eine Fraktion innerhalb des Medizinalkollegiums, die die Hamburger Psychiatrie für heillos rückständig hielt.

1897 gab es in Hamburg zwei öffentliche Anstalten. Die Irrenanstalt Friedrichsberg galt bei ihrer Gründung 1864 als eine der fortschrittlichsten im deutschsprachigen Raum. Ihr Leiter, Ludwig Meyer (1827-1900), hatte als einer der ersten das Non restraint eingeführt ${ }^{40}$. Er folgte 1866 einem Ruf nach Göttingen, Reye wurde sein Nachfolger. Während seiner Ägide, die 42 Jahre lang, bis 1908, währte, sah Hamburg ein massives Bevölkerungswachstum, dem eine Zunahme der Anstaltspopulation parallel ging. Um 1860 hatte Hamburg circa 175000 Einwohner. 1880 zählte man circa 450000, 1895 mehr als $690000^{41} .1864$ hatte Friedrichsberg Platz für 240 Patienten, 1880 war

38 Siehe dazu Rodegra 1979.

39 Siehe Schmiedebach 1996; Goldberg 2002, Goldberg 2003.

40 Zu Friedrichsberg: Weygandt 1922; zu Meyer: Burkhart 1991, zum Non restraint: Geduldig 1975.

41 Nipperdey 1993,113; Kraus 1882, 4; Reincke 1897, 1. 
die Anstalt mit 987 Patienten belegt, 1885 waren es 1082, 1890 1230. Erst die Gründung der «Landwirtschaftlichen Kolonie für Geisteskranke zu Langenhorn» am Nordrand Hamburgs im Jahr 1893, die für 200 chronische, rüstige, arme Patienten eingerichtet wurde, verschaffte kurzzeitig Luft: 1893 befanden sich 1095 Patienten in Friedrichsberg, 1895 1171, am 1. Januar 1898 versorgte Friedrichsberg 1351 Patienten $^{42}$.

Die Psychiatrie unter Reye galt als rückständig. Kritisiert wurde zum einen die mangelhafte ärztliche Versorgung. So mokierte sich 1894 der Physikus Carl Reinhard (1849-1896), 1883 bis 1889 zweiter Arzt in Friedrichsberg, es sei höchste Zeit gewesen, dass Reye eine weitere Arztstelle beantragt habe, so habe es nicht mehr weitergehen können ${ }^{43}$. Reincke selbst notierte 1897 , Friedrichsberg sei «seit Jahrzehnten die mit Ärzten am sparsamsten versorgte Anstalt Deutschlands» ${ }^{44}$. Tatsächlich war auf die Patientenzunahme in Friedrichsberg nicht mit einer Vermehrung der Arztstellen reagiert worden ${ }^{45}$, ein Zustand, der unter der Psychiatriekritik kaum tolerabel schien, wenn es um eine adäquate Versorgung und bessere Kontrolle des oft der Gewalttätigkeit verdächtigten Pflegepersonals ging. Zum zweiten machte man Reye für den Abbau des humanen Antlitzes der Anstalt verantwortlich. Die während seiner Zeit errichteten Erweiterungsbauten erhielten Eisengitter und erweckten in Fachkreisen den Eindruck von «Elefantenhäusern» ${ }^{46}$. Doch Reye behauptete sich. Das lag erstens daran, dass er sich «nach oben und nach unten den gebührenden Respekt zu verschaffen» ${ }^{47}$ wusste. Zweitens hielt er Hamburg geräuschlos eine Spielart devianten Verhaltens mit geringen Kosten vom Hals. Er fand hierbei eine Lobby im Krankenhauskollegium, dem für die Verwaltung der Krankenhäuser zuständigen Gremium, das nicht selten anderer Meinung war als das tendenziell eher mit medizinischem Fachverstand ausgestattete Medizinalkollegium.

42 Schnitzer 1901, 162; Jahrbücher 1893/94, 173; Jahrbücher 1897/98, 111. Zu Langenhorn: Kressin 1950.

43 Reinhard an Reincke, 16.7.1894, StHH 352-3. Medizinalkollegium II L 1: Gesetze und Verordnungen betr. das Irrenwesen in Hamburg Bd. 1 1846-1899, Bll. $37 f$.

44 Entwurf Reincke, 23.10.1897, StHH Bewerbungen, Bl. 55.

451889 arbeiteten in Friedrichsberg der als «Oberarzt» bezeichnete ärztliche Direktor, ein zweiter Arzt sowie drei «Gehülfsärzte», ein Zustand, der sich bis 1897 nicht änderte, erst jetzt wurden neben dem Direktor zwei Oberärzte, vier «Gehülfsärzte», zwei «diätarische Hülfsärzte» sowie ein Volontärarzt beschäftigt: vgl. Jahrbücher 1889, XXXVIII; Jahrbücher 1890, XVI; Jahrbücher 1891/92, XII, XVIII; Jahrbücher 1893/94, XIII, XIX; Jahrbücher 1895/96, XIII, XIX; Jahrbücher 1897/98, XIV, XX.

46 So die Aussage des alten Meyer, den Wahncau bei seiner Rückreise von Baden-Baden im Mai in Göttingen besuchte: Reisebericht Wahncau, 24.5.1897, StHH Bewerbungen, Bl. 40.

47 So Theodor Deneke in einem Nachruf auf Reye: StHH Reye Zeitungsausschnittsammlung A 767, Hamburger Correspondent Nr. 118, Morgenausgabe, 5.3.1912. 
Die Patientenzunahme führte schon 1894 zu Plänen einer Erweiterung Langenhorns auf 500 Betten, einer Umwandlung in eine Anstalt nach dem Modell des sächsischen Alt-Scherbitz (Zentralanstalt: geschlossene Häuser; Kolonie: ruhige, ungefährliche Kranke $\left.{ }^{48}\right)$. Der Antrag des Senats an die Bürgerschaft im April 1897 sah die Erweiterung Langenhorns, Neubauten in Friedrichsberg und Vermehrung des ärztlichen Personals vor. Intern, in einem Entwurf für das Medizinalkollegium vom Oktober $1897^{49}$, skizzierte Reincke ein Konzept, das die Neubesetzung dreier Stellen vorsah. In Friedrichsberg sollten unter dem Direktor zwei Oberärzte tätig, in Langenhorn, das formal Friedrichsberg unterstellt war, ein weiterer eingestellt werden. Diese Stellenbesetzungen müssten «aus einem Gusse erfolgen», davon hinge die Reorganisation des ärztlichen Dienstes ab. Die Oberärzte seien «Hauptträger» dieses Dienstes, sie müssten Verwaltungserfahrung mitbringen, mussten als psychiatrische Sachverständige vor Gericht tätig sein, schliesslich für die Assistentenausbildung und «die Pflege der wissenschaftlichen Psychiatrie» sorgen. Der Langenhorner Oberarzt sollte die erweiterte Anstalt, die auch unruhige Kranke aufnehmen sollte, neu organisieren. Diese Konzeption aus einem Guss war unabdingbar für reibungsloses Funktionieren: Langenhorn und Friedrichsberg waren eng verzahnt, Aufnahmen erfolgten weiterhin über Friedrichsberg, daher mussten alle Ärzte wechselseitige Kenntnis haben, um Konflikte zu vermeiden. Eine gleichzeitige Entscheidung über alle drei Stellen sei überdies wichtig, weil den Anstalten damit endlich «frisches Blut in genügender Menge» zugeführt werde. Reincke wollte Reyes Einfluss neutralisieren. Würde man nur eine Stelle in Friedrichsberg besetzen, so wäre die Wirkung des Neuberufenen zu gering. Für Langenhorn sah man einen Anstaltspraktiker vor, die Stelle in Friedrichsberg sollte «mit einer hervorragenden wissenschaftlichen [...] Kraft» besetzt werden ${ }^{50}$. Diese Arbeitsteilung war nicht von vornherein geplant. Bei den späteren Bewerbern nahm man auch Anstaltspsychiater wahr. Das Medizinalkollegium war zwar an Wissenschaft interessiert, doch das meinte nicht Avantgarde, sondern gediegen-vermittelnde Publikationen ohne Ecken und Kanten ${ }^{51}$. Nun, im Oktober, schlug Reincke ein Revirement vor. Der zweite Arzt Friedrichsbergs, Theodor Neuberger (1856-1938) ${ }^{52}$, sollte nach Langenhorn wechseln, der dort tätige Heinrich Schäfer (1857-?) ${ }^{53}$ könne sich

48 Siehe dazu differenzierter Paetz 1893.

49 Zum folgenden: Entwurf Reincke, 23.10.1897, StHH Bewerbungen, Bll. 52-56.

50 Protokoll Sitzung ärztliche Mitglieder Medizinalkollegium, 9.10.1897, StHH Bewerbungen, Bl. 51.

51 Siehe weiter unten.

52 Sammet 2005.

53 Kreuter 1996, III, $1237 f$. 
selbstverständlich auch bewerben, nach Auskunft Reyes zeigte er Interesse. Doch hielt man Schäfer im Medizinalkollegium für unfähig. Er besitze weder genügend Initiative noch Fähigkeiten, noch Interesse an der Weiterentwicklung Langenhorns. Seine Schriften erregten unter Fachgenossen «Befremden», kurz: Eine «vortheilhafte Entwicklung» der Anstalt unter ihm sei «unmöglich».

\section{John Wahncaus Begutachtungen im Mai und Juli 1897}

John Wahncau ${ }^{54}$ lieferte für das Medizinalkollegium die Expertise über die Kandidaten. 1862 in Hamburg geboren, promovierte er 1886 in Halle (Saale), war ab 1892 als Assistenzarzt am Krankenhaus St. Georg, zugleich als Polizeiarzt in St. Georg und Hammerbrook, seit 1893 als Physikus tätig. Nach dem Tod Reinhards, der aufgrund seiner psychiatrischen Erfahrung einschlägige Themen im Medizinalkollegium bearbeitet hatte ${ }^{55}$, übernahm Wahncau informell diese Rolle. Er fungierte als Vertrauter Reinckes ${ }^{56}$. Er konnte so, auf der Folie seines Habitus als Hamburger Bürger in Verwobenheit mit den skizzierten Spezifika des Hamburger Feldes als legitime Stimme, ausgestattet mit Klassifikationsmacht, Bewerber positionieren und in ihren wechselseitigen Relationen definieren. An den später aufgestellten Rubriken in einem Attributenkatalog lassen sich Eigenschaften ersehen, die ein Stellvertreter und zukünftiger Direktor mitbringen musste. Dieser komplexe Algorithmus, abgeleitet aus Alter, derzeitiger Stellung (als Hinweis auf professionelle/laufbahnspezifische Stellung im Spiel), Fachausbildung, Empfehlungen und Zeugnissen (mit Auflistung der Bürgen, falls vorhanden), Publikationen, Lehre, fungierte als Entscheidungshilfe. Hier zählte auch Leistung. Aber dieses Kriterium allein erklärt die Entscheidungsfindung nicht. Diese Attribute spiegelten aufgehäufte Kapitalien der Bewerber und die Wahrnehmung ihres Nutzens in Hamburg.

In seinem Reisebericht kritisierte Wahncau eine von ihm wahrgenommene «Kräpelin'sche Schule», Kraepelin (1856-1926) ${ }^{57}$ vertrete «seit jeher radicale Auffassungen», erwecke den «Eindruck eines einseitigen Fanatikers». Aus dessen Schule könne kaum ein für Hamburg passender Oberarzt

54 Zu Wahncau: StHH 111-1 Senat Cl. VII Lit. Lb No. 23a Vol. 88 Fasc. 8, 13, 18.

55 So geht zum Beispiel die Gründung Langenhorns als agrikole Kolonie auf seine Vorschläge zurück: siehe dazu Kressin 1950. Zur Biographie Reinhards: Kreuter 1996, III, $1164 f$.

56 Zum folgenden: StHH Bewerbungen, Reisebericht Wahncau, 24.5.1897, Bll. 36-40, sowie ebd., Gutachten Wahncau, 23.7.1897, Bll. 37-49 (die Blattzählung 37-40 ist zweimal vorhanden).

57 Die Literatur zu Kraepelin ist inzwischen Legion, in aller Kürze: Kreuter 1996, II, 762-766. 
kommen. «Passen〉 konnte nur ein schulenübergreifender Vertreter des common sense. Wahncau gefiel Max Dinkler (1863-1929) am besten, seit 1896 leitender Arzt am Luisenhospital in Aachen, doch dieser hatte nur Neurologisches publiziert ${ }^{58}$. Er schied daher aus, auch wenn Wahncau anmerkte, Dinkler sei Schüler Wilhelm Erbs (1840-1921). Hier spielte nicht nur Erbs Prestige als Begründer der Neurologie, sondern sein lokales symbolisches Kapital mit ${ }^{59}$. Sowohl Carl Eisenlohr (1849-1896), der 1887 bis 1896 eine internistische Abteilung im Neuen Allgemeinen Krankenhaus in Eppendorf geleitet hatte und, vorwiegend neurologisch orientiert, als einer der ersten Neurologen in Hamburg gelten konnte ${ }^{60}$, wie auch sein Nachfolger Max Nonne (1861-1959) ${ }^{61}$ konnten sich rühmen, Schüler Erbs gewesen zu sein; Nonne selbst hatte sich in seiner Heidelberger Zeit mit Dinkler angefreundet $^{62}$. Kennen und Anerkennen und das Prestige Erbs, das hier gewissermassen auf einen seiner Schüler abstrahlte, machten Dinkler sichtbar. Zwar wies sein Publikationsschwerpunkt Neurologie Wahncau darauf hin, dass er in zentralen Erfordernissen für die Hamburger Stelle nicht passte, doch zeigt sein Gesehenwerden, welchen Stellenwert eine, wenn auch indirekte, Bekannt- und Vertrautheit hätte haben können.

Dann sah sich Wahncau Alois Alzheimer (1864-1915) an. Dessen Laufbahnposition hätte gepasst, Wahncau sah ihn, er liess sich nicht darüber aus, was ihn in den Blick rückte. Doch lassen sich einige Angaben machen. Er wurde in Marktbreit in Bayern als Sohn eines Notars geboren, stammte also aus dem Honoratiorenbürgertum in kleinstädtischem Milieu. Das war kein schlechter Start, seine Primärsozialisation signalisierte schichtspezifische Ähnlichkeit. Seit 1888 war er, seit 1895 als Oberarzt, an der Anstalt für Irre und Epileptische in Frankfurt am Main tätig. Er war jung, 33 Jahre, hatte einiges publiziert, wenn auch eher Neuropathologisches, Erfahrungen mit Patienten und Verwaltung einer grossstädtischen Anstalt lagen vor ${ }^{63}$. Doch kam Alzheimer «seiner ausgesprochen süddeutschen Art wegen» nicht in Frage. Dialektale Sprechweise, Differenz im wahrgenommenen Verhalten,

58 Zu Dinkler: Kreuter 1996, I, 256.

59 Zu Erbs symbolischem Kapital innerhalb der Hagiographie als einer der Urväter der Neurologie: Nonne 1970.

60 Zu Eisenlohr und seiner Rolle bei der Entwicklung der Neurologie: Jolly 1896; Nonne 1914.

$61 \mathrm{Zu}$ Nonnes Biographie: Wulf 1999.

62 Cf. dazu Nonne 1971,38, 156, 157, 167.

63 Zu Alzheimer: Stimpert 1994; Niß1 1917; Gaupp 1916. Sicher gab es Unterschiede zwischen Frankfurt am Main und Hamburg, doch beide Städte hatten seit Beginn des Jahrhunderts ein rasantes Bevölkerungswachstum durchlaufen: Um 1800 hatte Frankfurt nur 48000 Einwohner, 1875 waren es etwas mehr als 100 000, um 1890 war diese Zahl auf knapp 180000 angestiegen (Nipperdey 1993, 113; Hohorst/Kocka/Ritter 1978, 45). 
kurzum: die regionale Färbung seines Habitus hätte auf sprödem Hamburger Parkett nicht funktioniert, minderte massiv sein kulturelles Kapital ${ }^{64}$.

Genauer liess sich Wahncau über Alfred Hoche aus. Er mache einen «angenehmen, frischen Eindruck», sei «verständig», scheine «Kenntnisse zu besitzen», erfreute sich grosser Beliebtheit in der Versammlung, mit einem Hamburger Schulrat gleichen Namens sei er nur entfernt verwandt - das war eine schöne Basis. Hoche hatte eine, wenn auch minimale, Anbindung an Hamburg. Seine «Frische» verhiess reformerischen Elan und Arbeitskraft, er funktionierte in der Zunft, warum sollte sein soziales Kapital der Beliebtheit nicht auch in Hamburg Vertrauen schaffen? Doch leicht beunruhigt notierte Wahncau, Hoche habe manchmal eine «etwas lose Zunge». Das musste nicht schlimm sein, markierte aber ein Risiko. Fast sämtliche Nekrologe betonen Hoches Hang zu sarkastischen Formulierungen, deren entschärftere Spielart seine «geistreiche» Art war ${ }^{65}$. Hier ging es nicht um Psychologie, sondern um eine Gefahr, die zugleich Problemlösungsressource sein konnte. Der Habitus als strukturierte, inkorporierte Geschichte ist generatives Schema der Praxis, er legt nicht einzelne Handlungen fest. Es verbleibt Spielraum, «unendlich» viele und «relativ unvorhersehbare Praktiken von dennoch begrenzter Verschiedenartigkeit ${ }^{66}{ }^{6}$ sind möglich. Vertrauen, das man in Hoche gesetzt hätte, hätte von ihm bestätigt oder missbraucht werden können. Seine «lose Zunge» hätte beide Male agiert. In Entscheidungs-, Aushandlungssituationen konnte Esprit ein Problem lösen, eine $z u$ lose Zunge als Teil des Hocheschen Habitus hätte aber Dysfunktionalität erzeugen können.

Ansonsten machte in Baden-Baden niemand Eindruck auf Wahncau. Von denjenigen, die in Hamburg als «in Betracht kommend» aufgefasst worden waren, sei keiner anwesend gewesen. Unter diesen neun erwähnten waren vier, die in Berlin arbeiteten oder gearbeitet hatten. Im Wahrnehmungsschema Hamburger Entscheidungsträger spielte die Strahlkraft der Charité zumindest bei dreien eine Rolle: Max Köppen (1859-1916), der in den 1890er Jahren ins Rampenlicht kam, aber durch eine neurologische Erkrankung

64 Zum Einfluss klassen- oder regionalspezifischer Sprechweise auf den Wert kulturellen Kapitals: Bourdieu 1992, 57. Dieses Moment der regional gefärbten «habitus» wurde später, bei den offiziellen Bewerbern, nochmals benutzt. Als sich der in Riedlingen/Württemberg 1853 geborene Paul Mayser um die Oberarztstelle bewarb, wurde, vor der Folie seines Alters, eine mögliche Inflexibilität gemutmasst: ob er sich mit 44 Jahren noch Hamburger Verhältnissen anpassen könne, nicht zuletzt, da er wegen seiner süddeutschen Abstammung dem norddeutschen «Wesen vielleicht fremdartig gegenüberstehen könnte». StHH Bewerbungen, B1. 59.

$65 \mathrm{Zu}$ Hoche und zu seiner «losen Zunge»: Gaupp 1943, Bumke 1943, Nonne 1943.

66 Bourdieu 1987, 104. 
am Ausbau seiner Karriere gehindert wurde ${ }^{67}$, war seit 1891 an der Charité. Wahncau kannte ihn von Vorträgen. Er hatte seine Zweifel an dessen Qualifikation. Er schien für die akademische Laufbahn gut geeignet zu sein, doch ob er «genügende practische Befähigung» entwickeln könne, schien ihm frag$\operatorname{lich}^{68}$. Hamburg benötigte zuallererst einen zupackenden Praktiker, jemand, der den Eindruck vermittelte, auch vor Verwaltungsmühen und der Fron täglicher Praxis nicht zu kapitulieren - Köppens inkorporiertes kulturelles Kapital, feinsinniges Gelehrtentum, als Teil seines Habitus, passte hier nicht. Justus Boedeker (1863-1936), über den sich Wahncau nicht weiter äusserte, war auch an der Charité unter Friedrich Jolly als Assistent tätig gewesen, später arbeitete er in Berlin-Herzberge unter Carl Moeli ${ }^{69}$. Robert Wollenberg (1862-1942), jetzt in Halle, hatte ebenfalls an der Charité gearbeitet. Bei Friedrich Wilhelm Kortum (1850-1928) beeindruckte nicht der universitäre Hintergrund, sondern die Tatsache, dass er in Hamburg bekannt war ${ }^{70}$. Von den weiteren waren Wahncau zwei persönlich bekannt: Albert Buchholz (1859-1927), jetzt in Jena, hatte er zwei Jahre zuvor in Marburg kennengelernt, August Cramer (1860-1912) ${ }^{71}$, Göttingen, besuchte er auf seiner Rückreise $^{72}$.Von Buchholz erhielt Wahncau «den besten Eindruck», er wurde ihm «von verschiedenen Seiten [...] als tüchtig bezeichnet». Besonders angetan war er von Cramer: «eine stattliche Erscheinung von angenehm geselligen Formen», er zeige «gediegene Kenntnisse, gesundes klares Urtheil und das, was man insgesamt gesunden Menschenverstand nennt, den man leider bei verschiedenen Richtungen der Psychiatrie neuerdings vermisst». Wahncau hielt ihn besonders deshalb für «hervorragend qualificirt», weil er, als Stellvertreter Meyers, «ausgezeichnete Qualitäten als Stütze eines alternden Chefs bewiesen» habe - eine attraktive Parallele zu Hamburg ${ }^{73}$.

Die Bewertungen Wahncaus zeigen, dass er eingebrachte Kapitalien und Erscheinungsbild der jüngeren Ärzte nach prospektivem Funktionieren in Hamburg beurteilte. Am sichersten war er, wenn ihm der Bewerber von

67 In einem Nekrolog hiess es, dass ihm das Leben «nicht erfüllt» habe, was es ihm nach seinen «wissenschaftlichen Leistungen und Erfolgen in den 90er Jahren [...] versprochen hatte»: B. 1916.

68 Reisebericht Wahncau, 24.5.1897, StHH Bewerbungen, Bl. 38.

69 Zu Boedeker: Kreuter 1996, I, 156f. - Zu Jolly und Moeli siehe weiter unten.

$70 \mathrm{Zu}$ Wollenberg und Kortum siehe weiter unten.

71 Zu Buchholz, der 1901 nach Hamburg wechselte: Kreuter 1996, I, 201; zu Cramer: Kreuter 1996, I, 234-236.

72 Daneben waren im Gespräch: ein Arzt Fischer aus Jena, zu dem keine weiteren Angaben eruiert werden konnten; Clemens Neisser aus Leubus, Max Krell aus Colditz (zu beiden siehe unten).

73 Reisebericht Wahncau, 24.5.1897, StHH Bewerbungen, Bll. 38f. - Weder Cramer noch Buchholz spielten allerdings bei dem nachfolgend skizzierten Auswahlverfahren eine Rolle, da sich keiner von beiden bewarb. 
Angesicht zu Angesicht oder über persönliche Kontakte anderer bekannt war und er Ähnlichkeiten wahrnahm. Das Fehlen eines spezifischen Habitusaspekts konnte isoliert zum Ausschluss führen. Alzheimers bayerische Wesensart minderte sein kulturelles Kapital, schmälerte aber zugleich sein prospektives soziales Kapital. Welche Entwicklung hätten Patientenbehandlung, Konflikte, Verhandlungen, Bewegungen auf gesellschaftlichem Parkett, kurz: sein soziales Netzwerk und Funktionieren nehmen können? Das war nicht vorhersehbar, Wahncau musste Risiko mindern. Im Falle Köppens war es eine spezifische Form inkorporierten kulturellen Kapitals, das negativ vermerkt wurde. Er war psychiatrisch geschult, doch für die praktische Anstaltspsychiatrie nicht tauglich. Ebenso fiel Dinkler durch, ihm fehlte gänzlich das inkorporierte Kapital psychiatrischer Erfahrung. Doch wird schon an dessen Beurteilung deutlich, dass Wahncau genau abzuwägen wusste. Zwar konstruierte er Ausschlusskriterien, aber betrachtete Habitusaspekte und nutzbare Kapitalien selten isoliert. Dinkler hatte er sich mit Rückgriff auf die Bekanntheit Erbs und seiner Schüler Eisenlohr und Nonne vertraut gemacht; funktionierten diese in Hamburg, so hätte auch jener einen Posten besetzen können. Doch spielte er im falschen Feld, die Psychiatrie war ihm zu fern. Bei Hoche war Wahncau vorsichtig. Er war unübersehbar interessant. Er war wohlausgebildet, er war klug, er war beliebt. Die Akkumulation vieler Kapitalien (psychiatrische Erfahrung, Verständigkeit im Fach, Anerkennung und Beliebtheit, sein Verhalten im Kontakt mit anderen) erschien Wahncau attraktiv - doch bestand nicht die Gefahr, dass sein soziales Netzwerk, sein Prestige in Hamburg durch seine lose Zunge schnell Schaden genommen hätten?

Im Juli positionierte Wahncau die anfangs 29, mit Nachmeldungen über 30 Bewerber. Zwei Anstaltspraktiker ohne universitären Hintergrund, die ihre Bewerbung zurückzogen, weil man ihnen zu Hause Angebote machte, hätten «nach Alter, Stellung und Erfahrung» in Frage kommen können. Gerhard Backenköhler (?-1923) ${ }^{74}$ war seit 1895 Direktor der westfälischen Provinzialanstalt Aplerbeck bei Dortmund. Er empfahl sich als Direktor einer Anstalt mit grossstädtischem Einzugsgebiet und durch die Tatsache, dass er unter Meyer in Göttingen gearbeitet (und dabei gleich dessen zweite Tochter geheiratet) hatte. Auch Adolf Stoltenhoff (?-1917) ${ }^{75}$ war schon Direktor, ein Praktiker, seit 1891 leitete er die Anstalt Kortau in Ostpreussen. Er hatte weiteres, soziales, Kapital zu bieten, er war von 1880 bis 1882 in Friedrichsberg beschäftigt gewesen. Beide waren erfahren in Verwaltung und 
Behandlung, waren keine «Anwärter» mehr, hatten Hamburg-Bezug, wenn auch bei Backenköhler nur indirekt; denn hier wirkte die legendenhafte Wertschätzung Meyers in Hamburg nach.

Wahncau teilte die Kandidaten in drei Gruppen. Vierzehn kamen nicht in Frage. Cruewell ${ }^{76}$, der sich als Biochemiker bezeichne, scheine seinen «Schriftstücken nach ein Phantast» zu sein - nichts konnte einem nüchternen Hamburger Bürger mehr missfallen, eine ästhetisch-geschmackliche Beurteilung, die zugleich die Kompetenz des Bewerbers in Zweifel zog, liess Cruewell in der Wahrnehmung Wahncaus unsichtbar werden. Der Arzt Froehlich aus Itzehoe, einer schleswig-holsteinischen Kleinstadt unweit Hamburgs, war als praktischer Arzt in einer Zementfabrik tätig. Er hatte somit keine psychiatrische Vorbildung. Beide gehörten genaugenommen nicht zum psychiatrischen Feld und konnten ausgeschlossen werden. Ein dritter, Christian Roller (?-1897) $)^{77}$, gehörte zum Spiel, hatte sich aber selbst ins Aus gespielt, ohne gegen Regeln des psychiatrischen Felds verstossen zu haben. Er war erfahren, von 1884 bis 1896 war er Direktor der Anstalt Lindenhaus. Er war der Sohn Christian Friedrich Wilhelm Rollers (1802$1878)^{78}$, einem der Doyens der deutschsprachigen Psychiatrie, der mehrfach als Berater bei der Planung einer eigenständigen Irrenanstalt in Hamburg fungiert hatte ${ }^{79}$. Doch hatte sich Roller junior durch eine Liaison mit einer fragwürdigen Dame «gesellschaftlich unmöglich gemacht» ${ }^{80}$ - Ästhetisch-Ethisches in Wahncaus Denkschema gab den Ausschlag, ohne dass hier überhaupt noch andere Kapitalformen in Betracht gezogen werden mussten.

Neun Bewerber waren zu jung, ihnen fehlte Erfahrung zum Stellvertreter, keiner war publizistisch sichtbar genug. Dabei war nicht nur das biologische Alter gemeint, sondern eine Kombination aus Laufbahnalter (als Folge von Stringenz des Durchlaufens der Positionen vom Studium bis zum «Anwärter») und Verweildauer in der Psychiatrie. Erfahrung als inkorporiertes kulturelles Kapital umfasste nicht nur Geübtheit als Anstaltspsychiater. Unausgesprochen floss ein, ob ein so junger Arzt denn auch eine Stütze für den alternden Reye (und zugleich ein Gegengewicht gegen ihn) bilden konnte. Hier überzeugte Wahncau keiner vom Vorhandensein nötiger Kapi-

76 Es scheint sich um (Ernst) Rudolf Cruewell (1850-?) zu handeln, der als Homöopath tätig war: Wrede 1898.

77 Zu Christian Roller: Kreuter 1996, III, 1192.

78 Zu Christian Friedrich Wilhelm Roller: Fischer 1921.

79 Siehe zum Beispiel sein Gutachten über den Bau einer eigenständigen Anstalt von März 1841 oder seine späteren Stellungnahmen: Schmidt 1974, 22-30,33-38.

80 So Wahncaus Formulierung: StHH Bewerbungen, Bl. 47. 
talien, weder Adolf Dannemann (1867-1932) ${ }^{81}$, Johannes Bresler (18661942) ${ }^{82}$, Emil Bratz (1868-1934) ${ }^{83}$, Oskar Kluge (1865-1930) ${ }^{84}$, Paul Kemmler (1865-? $)^{85}$ noch ein Arzt Maas, der in einer Pankower Nervenheilanstalt tätig war, oder ein Arzt Orthmann aus Düsseldorf oder zwei Assistenten aus Berlin-Dalldorf ${ }^{86}$.

Gesondert betrachtete Wahncau drei mit Hamburg verbundene Ärzte. Carl Adolf Passow (?-1920) ${ }^{87}$ hatte zwar als «Gehülfsarzt» in Friedrichsberg gearbeitet, doch das genügte nicht, er war noch kein Anwärter. Alfred Boettiger, in Nervenkrankheiten spezialisierter Arzt aus Hamburg, konnte keine Distinktionsgewinne erzielen, er sei sehr jung, habe keine «psychiatrische Abtheilung selbständig geleitet». Sein symbolisches Kapital war gleich null, er galt «in Psychiaterkreisen, welche ihn von seiner Assistentenzeit her kennen, für unbedeutend ${ }^{88}$. Ausführlicher ging Wahncau auf Theodor Kaes (1852-1913) ein, seit 1890 in Friedrichsberg. Er käme seinem Alter nach in Frage, sei «ein fleissiger, wissenschaftlich strebender und gelehrter Arzt, der sich durch seine gehirnanatomischen Arbeiten einen Ruf» erworben habe, doch fehle ihm die «nöthige Sicherheit und Gewandtheit des Auftretens», die von einem Oberarzt «unbedingt zu verlangen sind» ${ }^{89}$ - Wahncau verteilte die nicht passenden Bewerber nach heterogenen Kriterien im Feld. Zwei kamen nicht in Frage, weil sie überhaupt nicht im psychiatrischen Feld mitspielten. Ein dritter hatte gegen ungeschriebene moralische Gesetze verstossen. Zentrale Bewertungskriterien für die weiteren elf Bewerber, die schnell ausgeschlossen wurden, waren für Wahncaus Wahrnehmungs- und Denkschemata deren (fehlendes) institutionalisiertes und inkorporiertes kulturelles Kapi-

81 Er war 31 Jahre alt, hatte keine geradlinige Laufbahn. Zuerst hatte er Geschichte und Literaturwissenschaft studiert, dann wechselte er zur Medizin. Bis 1894 fuhr er als Schiffsarzt zur See, ehe er in die Bremer Irrenanstalt als Assistent eintrat, 1896 kam er an die Psychiatrische Universitätsklinik Giessen. Er war seit drei Jahren im Feld, drei Publikationen lagen vor. Cf. Sommer 1932; Meltzer 1932.

82 Bresler war 31 Jahre alt, seit sechs Jahren in der Anstaltspsychiatrie, seit 1895 Oberarzt in Freiburg/Schlesien, einige wenige Publikationen. Vgl. Weygandt 1936.

83 Bratz war erst 29 Jahre alt, er war seit sechs Jahren in Anstalten beschäftigt, doch hatte er in der Epileptikeranstalt Berlin-Wuhlgarten nur Assistentenstatus, eine Publikation. Vgl. Panse 1934.

84 Kluge war 32 Jahre alt, als Kapital konnte er seinen Volontärarztstatus ins Feld führen, publiziert hatte er noch nichts. Vgl. Gallus 1931.

85 Kemmler war 32 Jahre alt, seit sechs Jahren psychiatrisch tätig, seit 1895 arbeitete er als Sekundärarzt im württembergischen Zwiefalten, zwei Publikationen. Vgl. Kreuter 1996, II, 703.

86 Präzisere Angaben zu Maas und Orthmann und den Dalldorfer Ärzten liessen sich nicht eruieren.

87 Zu Passow: Kreuter 1996, III, 1076; Weygandt 1922, 6.

88 StHH Bewerbungen, Bl. 49.

$89 \mathrm{Zu}$ Kaes: Weygandt 1914, dort auch die Liste seiner neuroanatomischen Arbeiten. StHH Bewerbungen, B1. 59. 
tal. Doch verrechnete er dies mit deren möglichen Handlungs- und Verhaltensoptionen in Hamburg. Ihnen fehlte so die Stellung, sich in Hamburg zu behaupten, soziales und symbolisches Kapital anzuhäufen. Vorentscheidend war hier, wer wann welche Position durchlaufen und wer im Vergleich mit anderen überhaupt schon den Anwärterstatus erreicht hatte.

Hier folgten nun vier für wesentlich gleichwertig Erachtete. Zuerst Friedrich Wilhelm Kortum ${ }^{90}, 48$ Jahre alt, seit 15 Jahren psychiatrisch tätig. Er hatte 1883 bis 1885 in Friedrichsberg gearbeitet, bis 1893 fungierte er als ärztlicher Direktor der Pflegeanstalt Merxhausen bei Kassel, dann wechselte er als Oberarzt nach Berlin-Herzberge. Das war nicht nur eine Laufbahn comme il faut, man hatte Kenntnis, wie er in Hamburg funktionierte. Er hatte überdies eine Empfehlung seines Chefs. Er kenne sich in Administration und forensischer Psychiatrie aus. Seine Positionierung im Bewerberfeld war mithin eine komplexe Funktion seiner lokalen Bekanntheit, seiner Laufbahnstufe, der spezifischen Färbung seines inkorporierten kulturellen Kapitals, als ärztlicher Direktor verfügte er über eine Fülle von praktischer Verwaltungserfahrung und hatte forensisch begutachtet. Überdies zehrte er vom symbolischen Kapital seines Bürgen und Chefs Karl Moeli (1849-1919), der selbst durch seine Laufbahn beeindruckte und psychiatriepolitisch um 1900 der erste im Feld war, so dass später von ihm gesagt wurde, kein anderer Psychiater habe solch massgeblichen «Einfluss auf die neuere Entwicklung des Irrenwesens in Preussen gehabt ${ }^{91}-$ und nicht nur dort. Man kannte sich gut, der Hamburger Senat hatte sich 1896 an Moeli mit der Bitte um ein Gutachten über den Ausbau Langenhorns gewandt ${ }^{92}$, die Bande des Vertrauens waren eng, man konnte sich auf das Urteil Moelis verlassen.

Neben Kortum sah Wahncau Neuberger als gleichwertig an. Dessen «langjährige Thätigkeit in Friedrichsberg und dessen reges Interesse an der Fortentwicklung dieser Anstalt» hatte «allseitig Anerkennung gefunden». Für ihn sprachen die lokale Vertrautheit und Verankerung, später die spezifische Stellung, die ihm zugedacht war. Er sollte dann ja die praktische Seite der Versorgung in einer «Chroniker»-Anstalt, Langenhorn, übernehmen. Er passte gut. Er war 41 Jahre alt, von 1882 bis 1885 war er Assistent der Osnabrücker Anstalt gewesen und wechselte dann nach Hamburg. Er war

$90 \mathrm{Zu}$ Kortum: Kreuter 1996, II, 760.

91 So Liepmann 1919, 526. - Seit 1880 war Moeli Assistent bei Westphal an der Charité gewesen. Er habilitierte sich, wurde 1884 Oberarzt, 1887 dirigierender Arzt Dalldorfs und ausserordentlicher Professor. 1893 übernahm er die Leitung Herzberges. Im selben Jahr wurde er Mitglied der wissenschaftlichen Deputation für das Medizinalwesen, Hilfsarbeiter im Kultusministerium, später dort Referent für das Irrenwesen. Vgl. Liepmann 1919; Henneberg 1919.

92 Siehe dazu Kressin 1950, 15; Schmidt 1974, 106f., das Gutachten Moelis ist ebd., 167-172, abgedruckt. 
also wie Kortum seit 15 Jahren in Anstalten, wissenschaftlich war er nicht aufgefallen, aber das musste nicht entscheidend sein, der Schwerpunkt konnte auf der Vertrautheit mit der praktischen Psychiatrie liegen.

Als dritter wurde Max Krell (1855-1912) ${ }^{93}$, 42 Jahre alt, erwähnt, sein ehemaliger Chef Adolf Knecht (1846-1915) ${ }^{94}$ empfahl ihn warm. Seine Karriere hatte Krell 1880 in der Privatanstalt des geschätzten Caspar Max Brosius (1825-1910) ${ }^{95}$ in Bendorf/Koblenz begonnen (und dabei dessen Tochter geheiratet). Dann wurde er Hilfsarzt in Colditz, wechselte nach Hubertusburg, schliesslich war er Oberarzt in Colditz. Dieser praktisch geschulte Mann, der durch seinen Benimm (er wurde als lebensfroh und als «brillanter Gesellschafter» beschrieben) einnahm, blickte auf 17 Jahre Anstaltstätigkeit zurück, einige Aufsätze rundeten das Bild ab.

Besonders gelobt wurde Robert Wollenberg (1862-1942) ${ }^{96}$. Das war nicht verwunderlich, er war nicht nur praktisch erfahren, sondern hatte auch wissenschaftliche Meriten vorzuweisen. Er war 35 Jahre alt, seit zwölf Jahren in der Psychiatrie, Oberarzt, ausserordentlicher Professor. Überdies besass er kaum schlagbares symbolisches Kapital. Ihm werde eine «führende Stelle in der modernen Psychiatrie allgemein und unbestritten zuerkannt», Autoritäten wie Eduard Hitzig (1838-1907), Friedrich Jolly (1844-1904), Heinrich Laehr (1820-1905) ${ }^{97}$ und Moeli (man hatte sich vertraulich erkundigt) bezeichneten ihn «als eine[n] der besten unter den jüngeren psychiatrischen Forschern». Kurz: «ein vielseitig gebildeter Irrenarzt und ein fleissiger, verständig kritischer wissenschaftlicher Forscher». Er mache persönlich einen «gediegenen und gewandten Eindruck». Aus dieser Eloge lässt sich erstens entnehmen, dass ihn nicht nur eine Fraktion im psychiatrischen Feld empfahl. Hitzig war durch seine Arbeiten über die elektrische Erregbarkeit der Hirnrinde ausgewiesen, Jolly, Chef der Nervenklinik der Charité, genoss hohes Prestige im universitären Feld, eine Empfehlung aus der Charité verbürgte in Hamburg Güte. Moeli deckte den Bereich Irrenwesen ab, Laehr war der Nestor der Anstaltspsychiatrie. Zweitens flösste Wollenbergs Werdegang Vertrauen ein. 1884, mit 22 Jahren, medizinisches Staatsexamen, 1885 Promotion in Leipzig, von 1885 bis 1888 war er Assistent in Halle-Nietleben gewesen, von 1888 bis 1891 arbeitete er bei Westphal an der Charité, 1892 holte ihn Hitzig nach Halle, er hatte Erfahrung in forensischer Begutachtung,

93 Zu Krell: Näcke 1913; Ilberg 1912/13.

94 Zu Knecht: Kreuter 1996, II, 728f.

95 Zu Brosius: Kreuter 1996, I, 190-193.

96 Zu Wollenberg: Drachler 1995.

97 Zu Hitzig: Kreuter 1996, II, 581-584; Laehr: Kreuter 1996, II, 812f.; Jolly: Kreuter 1996, II, 665-668. 
Anstalts- wie Universitätspsychiatrie waren ihm geläufig. Seine Schriften zeigten keine Einseitigkeiten, er lieferte kritisch-abwägende Arbeiten (ohne «radikal einseitig» zu sein), eben: common sense. Schliesslich passte sein Charakter. «Gediegen» zu sein, das meinte ${ }^{98}$ Tüchtigkeit, Reife in Verhalten und Urteil. Diese Konnotationen des in sich Ruhenden wurden ergänzt durch «Gewandtheit»: Geschicklichkeit, Flexibilität, Schmiegsamkeit des Geistes, des Verhaltens «gegenüber den Lebensverhältnissen»" - eine Eigenschaft, die zum Beispiel Kaes abging.

Drei weitere Kandidaten standen den vorgenannten nach. Anton Delbrücks (1862-1944) als übertrieben eingeschätztes «Temperenzlertum» signalisierte Einseitigkeit, seine Schriften wurden nicht allseits geschätzt. Doch hatte er laufbahnspezifisch einiges zu bieten - zum Beispiel eine zweijährige Assistententätigkeit in Friedrichsberg von 1888 bis 1890. Dann war er unter Auguste Forel (1848-1931) am Zürcher Burghölzli gewesen (woher sein radikaler Antialkoholismus resultierte), er hatte sich 1891 an der Universität Zürich habilitiert ${ }^{100}$. Alexander Westphal hatte, neben seinem Versuch, Reincke zu treffen, auch Empfehlungen von Jolly und Moeli vorzuweisen. Doch schien er, so Wahncau, im Vergleich zu Kortum noch nicht soweit. Er war 34 Jahre alt, 1888 ging er zu Erb nach Heidelberg. Anschliessend war er bei dem Internisten Heinrich Curschmann (1846-1910) in Leipzig - in Hamburg kein Unbekannter, war dieser doch die treibende Kraft beim Bau des Eppendorfer Krankenhauses gewesen ${ }^{101}$. Westphal wechselte an die Charité zu Jolly, wo er sich 1894 habilitierte. Er war kaum von seinem biologischen Alter her zu jung, denn er war nur wenig jünger als der hochgelobte Wollenberg, doch fehlte noch psychiatrische Erfahrung, überdies war Wollenbergs relationale Positionierung im Bewerberfeld durch seine Bürgen und durch die Bekanntheit seiner Charakterzüge besser. Schliesslich kam Clemens Neisser (1861-1940) ${ }^{102}$ in den Blick. Er hätte gute Chancen haben müssen. Er war 36 Jahre alt, seit mehr als zehn Jahren in der Psychiatrie, zuerst Assistent, dann Oberarzt unter Wilhelm Alter (1843-1918) in Leubus ${ }^{103}$. Seine Schriften empfahlen ihn als Praktiker und klinischen Wissenschaftler ${ }^{104}$. Richard Deutschmann (1852-1935) ${ }^{105}$, Ophthalmologe und leitender

98 Cf. Grimm/Grimm 1878.

99 So die Formulierung in Grimm/Grimm 1911, Sp. 5314.

100 Zu Delbrück: Kreuter 1996, I, 247f.; Weygandt 1922, 6. Zu Forel: Kreuter 1996, I, 368-732.

$101 \mathrm{Zu}$ Heinrich Curschmann, der von 1879 bis 1888 in Hamburg tätig war, cf. Weisser/Uhlmann 1989, 13-34.

102 Zu Neisser: Kreuter 1996, II, 1022f.; Bresler 1926.

$103 \mathrm{Zu}$ Alter, der die Anstalt Leubus in Schlesien von 1884 bis 1912 leitete: Kreuter 1996, I, 29-31.

104 So zum Beispiel seine Arbeit zur Katatonie: Neisser 1887.

$105 \mathrm{Zu}$ Deutschmann: Anonym 2002. 
Arzt der Augen-Poliklinik des Israelitischen Krankenhauses in Hamburg, empfahl ihn als «feine[n], liebenswürdige[n] College[n]», Alter betonte seine «geistige Regsamkeit, seine allgemeine Bildung, sein reiches medicinisches Wissen, seine hervorragende klinische Beobachtungsgabe». Wahncau sah ihn, nicht zuletzt seine Arbeiten zu klinischen und alltagspraktischen Themen. Nach der Papierform war er ein Kandidat, er kam in die engere Wahl. Doch wurde in den Diskussionen im Medizinalkollegium bemerkt, man könne nicht beurteilen, ob er sich von seiner Art her eigne, er sei niemandem persönlich bekannt.

Diese fehlende persönliche Bekanntheit, die Vertrauen abgesichert hätte, spielte in der dritten Gruppe die entscheidende Rolle, Wahncau stufte sie herunter, er kannte sie nur aus ihren Bewerbungen, die sie nicht sichtbarer machten: zu niedrige Laufbahnstufe, kaum Veröffentlichungen, kein universitärer Hintergrund, keine hochkarätigen Empfehlungen. Oder sie passten in Relation zu anderen Bewerbern nicht besser. Friedrich Buddeberg (?-1927), Oberarzt im rheinischen Merzig, war publizistisch kaum sichtbar, ebensowenig Johannes Dinter (1862-1937), Oberarzt im schlesischen Rybnik, oder Bernhard Schauen (?-1936) aus dem westpreussischen Conradstein ${ }^{106}$, ein Arzt Becker aus Neuruppin, E. Möller, Oberarzt in Plagwitz, ein Arzt Teichert aus Owinsk ${ }^{107}$. Was Max Tippel (1860-1912) an Kapital zu bieten hatte, war in Hamburg vertrauter erhältlich. Er war 37 Jahre alt, seit 1896 dirigierender Arzt der Irrenabteilung der Kaiserswerther Anstalten. Von 1886 bis 1894 hatte er in Alt-Scherbitz gearbeitet - nach diesem Modell sollte ja Langenhorn strukturiert werden. Doch Wahncau musste nicht nachdenken, Neuberger stand bereit, Tippel kannte er nicht persönlich ${ }^{108}$.

\section{Schluss}

Einleitend waren als Ziele dieser Skizze die Beschreibung eines Teilaspekts der Hamburger Psychiatriegeschichte und die probatorische Verwendung einiger soziologischer «Werkzeuge» genannt worden.

Funktionierte nun die Besetzung der Oberarztstellen so, wie es sich Wahncau, Reincke und das Medizinalkollegium vorstellten? Nein. Zwar versuchte man, Reye soweit als möglich aus der Wahl herauszuhalten. Doch wusste er sich zu helfen. Bei der Sitzung der ärztlichen Mitglieder des Medizinal-

106 Zu Buddeberg: Kreuter 1996, I, 202; zu Dinter: Kreuter 1996, I, 256f.; zu Schauen: Kreuter 1996, III, 1252.

107 Nähere Angaben zu Becker, Möller und Teichert konnten nicht eruiert werden.

108 Zu Max Tippel: Kreuter 1996, III, 1463; Herting 1913. 
kollegiums am 9. Oktober 1897 plädierte er für Neuberger und Kortum als neue Oberärzte, zwei Männer, die unter ihm gedient hatten, er versuchte in seinem Umfeld ihm Vertraute zu installieren. Dann musste er den Sitzungsraum verlassen, man besprach die Bewerber ohne ihn ${ }^{109}$. Im Krankenhauskollegium machte er seinen Einfluss dann geltend. Als dort am 14. Dezember 1897 gewählt wurde, entfielen auf Neuberger als Oberarzt in Langenhorn 8 Stimmen. Für Friedrichsberg kamen 9 Stimmen auf Heinrich Schäfer, 7 auf Wollenberg, nur 2 auf Kortum ${ }^{110}$. Der vom Medizinalkollegium als unfähig erachtete Schäfer wechselte als Oberarzt nach Friedrichsberg. Wollenberg kam im April 1898 mit grossen Hoffnungen. Friedrichsberg machte einen «gefängnisartigen Eindruck und schrie» nach Reformen: «Man wollte auch reorganisieren, dabei aber doch nicht sehr in die Tiefe gehen.» Wollenberg legte einen Reorganisationsplan vor, der in der Schublade verschwand. 1901 ging er nach Tübingen. Das hatte nicht nur mit Enttäuschung über die zähe Hamburger Psychiatrie, sondern wiederum mit der Frage einsetzbarer Kapitalien und Hoches «Naturgesetz» zu tun. Tübingen bot die Möglichkeit, wieder in die renommiertere akademische Laufbahn zu wechseln. Reye liess sich partout nicht pensionieren. In Hamburg, so Wollenberg, habe er «doch nur unsichere Zukunftsaussichten» gehabt ${ }^{111}$. Sicher verzögerte sich so der Generationswechsel in der Hamburger Psychiatrie.

Das Auswahlverfahren zeigt schliesslich die komplexe Verschränkung von individuellem Bewerberprofil, dem Habitus der Bewertenden in Hamburg sowie den Anforderungen im psychiatrischen wie im spezifischen Hamburger Feld. Es gab keinen einfachen Algorithmus in der Taxierung der Bewerber. Wahncau formulierte zwar bei einigen Aspiranten eindeutige Ausschlusskriterien, in der Gewichtung spielte die persönliche Bekanntheit als Positivum eine erhebliche Rolle, doch erst die Kombination aus den wahrgenommenen «habitus» als Funktion der am Markt einsetzbaren Kapitalien und die relationale Gewichtung jedes Bewerbers zu seinen Konkurrenten auf der Folie der Hamburger Erfordernisse machen die jeweilige Beurteilung plausibel.

Partiell blieb meine Analyse schematisch. Inwieweit das Bourdieusche Instrumentarium selbst problematisch ist, müssten methodologische Untersuchungen zeigen. Ausserdem müssten Kollektivbiographien statistische Daten liefern, die eine differenziertere sozialhistorische Einordnung erlaub-

109 Protokoll Sitzung ärztliche Mitglieder Medizinalkollegium, 9.10.1897, StHH Bewerbungen, Bl. 51.

110 Protokoll Sitzung Krankenhauskollegium, 14.12.1897, StHH Gesundheitsverwaltung Personalakten 76, Theodor Neuberger, B1. 1.

111 Wollenberg 1931, 93, 100. 
ten. Allerdings kann die Verwendung Bourdieuscher Begriffe helfen, spezielle Rekrutierungssituationen verständlicher $\mathrm{zu}$ machen. Weitere Forschungen müssen zeigen, wie gross die Reichweite eines solchen Zugriffs dann jenseits einer einzelnen Fallstudie sein könnte.

\section{Archivalien}

Staatsarchiv Hamburg:

111-1 Senat Cl. VII Lit. Lb No. 23a Vol. 88, Fasc. 8, 13, 18 John Wahncau

352-3 Medizinalkollegium I H 3 c, Begutachtung der Bewerbungen um Arztstellen bei den staat-

lichen Irrenanstalten, 1858-1920

352-3 Medizinalkollegium II L 1, Gesetze und Verordnungen betr. das Irrenwesen in Hamburg

Bd. 1 1846-1899

Wilhelm Reye Zeitungsausschnittsammlung A 767

352-10 Gesundheitsverwaltung Personalakten 76 Neuberger, Theodor

\section{Literatur}

Anonym, «Deutschmann, Richard», in: Dietrich von Engelhardt (Hrsg.), Biographische Enzyklopädie deutschsprachiger Mediziner, Bd. 1 (München 2002) 123f.

B(onhoeffer, Karl?), «Max Köppen †», Monatsschrift für Neurologie und Psychiatrie 39 (1916) 258

Bourdieu, Pierre, Die feinen Unterschiede. Kritik der gesellschaftlichen Urteilskraft (Frankfurt a.M.1982)

- Sozialer Raum und «Klassen». Leçon sur la leçon. Zwei Vorlesungen (Frankfurt a.M. 1985)

- Sozialer Sinn. Kritik der theoretischen Vernunft (Frankfurt a.M. 1987)

- Satz und Gegensatz. Über die Verantwortung der Intellektuellen (Frankfurt a.M. 1989)

- «Ökonomisches Kapital - Kulturelles Kapital - Soziales Kapital», in: Pierre Bourdieu, Die verborgenen Mechanismen der Macht. Schriften zu Politik und Kultur 1 (Hamburg 1992) 49-79

- Soziologische Fragen (Frankfurt a.M. 1993)

- Die Regeln der Kunst (Frankfurt a.M. 1999)

- /Martin De Saint, «Le patronat», Actes de la recherche en sciences sociales 20/21 (1978) 2-82

- /Jean-Claude Chamboredon/Jean-Claude Passeron, Soziologie als Beruf. Wissenschaftstheoretische Voraussetzungen soziologischer Erkenntnis (Berlin/New York 1991)

Bresler, J., «Clemens Neisser zum vierzigjährigen Dienstjubiläum», Psychiatrisch neurologische Wochenschrift 28 (1926) 205-211

Bumke, Oswald, «Alfred Erich Hoche $\dagger »$, Archiv für Psychiatrie und Nervenkrankheiten 116 (1943) 339-346

Burkhart, Elisabeth, Ludwig Meyer (1827-1900) - Leben und Werk. Ein Vertreter der deutschen Psychiatrie auf ihrem Weg zur medizinisch-naturwissenschaftlichen Fachdisziplin (Diss. med. Berlin 1991)

Dierse, Barbara, Carl Westphal (1833-1890) - Leben und Werk: Vertreter einer deutschen naturwissenschaftlich orientierten Universitätspsychiatrie im 19. Jahrhundert (Diss. med. Greifswald 1995)

Drachler, Christine Johanna, Robert Wollenberg. Leben und Werk (Diss. med. Tübingen 1995)

Evans, Richard J., Tod in Hamburg. Stadt, Gesellschaft und Politik in den Cholera-Jahren 18301910 (Reinbek 1990)

Fischer, Max, «Christian Friedrich Wilhelm Roller (1802-1878)», in:Theodor Kirchhoff (Hrsg.), Deutsche Irrenärzte, Bd. 1 (Berlin 1921) 189-201 
Gallus, Kurt, «Nekrolog Kluge», Allgemeine Zeitschrift für Psychiatrie 94 (1931) $465 f$.

Gaupp, Robert, «Alois Alzheimer †», Münchner Medizinische Wochenschrift 63 (1916) 195-197

- «Alfred Erich Hoche $\dagger »$, Zeitschrift für die gesamte Neurologie und Psychiatrie 176 (1943) $1-6$

Geduldig, Cordula, Die Behandlung von Geisteskranken ohne mechanischen Zwang: Die Rezeption des Non-restraint im deutschen Sprachgebiet (Diss. med. Zürich 1975)

Goldberg, Ann, «The mellage trial and the politics of insane asylums in Wilhelmine Germany», Journal of Modern History 74 (2002) 1-32

- "A Reinvented Public: 'Lunatics' Rights' and Bourgeois Populism in the Kaiserreich", German History 21 (2003) 159-182

Grimm, Jakob/Wilhelm Grimm, «Gediegen», in: Jakob Grimm/Wilhelm Grimm, Deutsches Wörterbuch, Bd. 4 (Leipzig 1878) Sp. 2020-2024.

- «Gewandtheit», in: Jakob Grimm/Wilhelm Grimm, Deutsches Wörterbuch, Bd. 6 (Leipzig 1911), Sp. 5312-5315

Hartmann, Michael, «Klassenspezifischer Habitus oder exklusive Bildungstitel als soziales Selektionskriterium? Die Besetzung von Spitzenpositionen in der Wirtschaft», in: Beate Krais (Hrsg.), An der Spitze. Von Eliten und herrschenden Klassen (Konstanz 2001) 157-208

- «Leistung oder Habitus? Das Leistungsprinzip und die soziale Offenheit der deutschen Wirtschaftselite», in: Uwe H. Bittlingmeyer/Rolf Eickelpasch/Jens Kastner/Claudia Rademacher (Hrsg.), Theorie als Kampf? Zur politischen Soziologie Pierre Bourdieus (Opladen 2002) 361-377

Henneberg, Richard, «Carl Moeli †», Neurologisches Centralblatt 38 (1919) 767f.

Herold-Schmidt, Hedwig, «Ärztliche Interessenvertretung im Kaiserreich 1871-1914», in: Robert Jütte (Hrsg.), Geschichte der deutschen Ärzteschaft. Organisierte Berufs- und Gesundheitspolitik im 19. und 20. Jahrhundert (Köln 1997) 43-95

Herting, Johannes, «Nachruf Max Tippel», Psychiatrisch neurologische Wochenschrift 14 (1913) 509-511

Hoche, Alfred, Jahresringe. Innenansichten eines Menschenlebens (München/Berlin 1941)

Hohorst, Gerd/Jürgen Kocka/Gerhard A. Ritter, Sozialgeschichtliches Arbeitsbuch II. Materialien zur Statistik des Kaiserreichs 1870-1914 (München 21978)

Ilberg, Georg, «Julius Alexander Max Krell $\dagger$ », Psychiatrisch neurologische Wochenschrift 14 $(1912 / 13) 405 f$.

Jahrbücher der hamburgischen Staatskrankenanstalten 1 (1889)

Jahrbücher der hamburgischen Staatskrankenanstalten 2 (1890)

Jahrbücher der hamburgischen Staatskrankenanstalten 3 (1891/92)

Jahrbücher der hamburgischen Staatskrankenanstalten 4 (1893/94)

Jahrbücher der hamburgischen Staatskrankenanstalten 5 (1895/96)

Jahrbücher der hamburgischen Staatskrankenanstalten 6 (1897/98)

Jolly, Friedrich, «Dr. C. Eisenlohr †», Archiv für Psychiatrie und Nervenkrankheiten 29 (1896) $343 f$.

Kirstein, Ruth Priscilla, Alfred Erich Hoche (1865-1943) in Straßburg (Diss. med. Freiburg 1997)

Krais, Beate/Gunter Gebauer, Habitus (Bielefeld 2002)

Kraus, Caspar Theodor (Hrsg.), Bericht des Medicinal-Inspectorats über die medicinische Statistik des Hamburgischen Staates für das Jahr 1881 (Hamburg 1882)

Kressin, Albrecht, Das allgemeine Krankenhaus Langenhorn in Hamburg (Hamburg 1950)

Kreuter, Alma, Deutschsprachige Neurologen und Psychiater. Ein biographisch-bibliographisches Lexikon von den Vorläufern bis zur Mitte des 20. Jahrhunderts (München/New Providence/London/Paris 1996)

Liepmann, Hugo, «Karl Moeli zum 70. Geburtstage», Deutsche medizinische Wochenschrift 45 (1919) 526f.

Luhmann, Niklas, Vertrauen. Ein Mechanismus der Reduktion sozialer Komplexität (Stuttgart 21973)

Meltzer, Ewald, «Heinrich Adolf Dannemann $\dagger »$, Psychiatrisch neurologische Wochenschrift 34 (1932) 349-352

Näcke, Paul, «Nekrolog Krell», Allgemeine Zeitschrift für Psychiatrie 70 (1913) $182 \mathrm{f}$.

Neisser, Clemens, Ueber die Katatonie. Ein Beitrag zur klinischen Psychiatrie (Stuttgart 1887) 
Nipperdey, Thomas, Deutsche Geschichte 1800-1866. Bürgerwelt und starker Staat (München 61993)

Nißl, Franz, «Zum Andenken A. Alzheimers», Allgemeine Zeitschrift für Psychiatrie 73 (1917) 96-107

Nonne, Max, «Carl Eisenlohr», in: Brauer, Ludolf (Red.), Festschrift dem Eppendorfer Krankenhause zur Feier seines 25jährigen Bestehens gewidmet (Leipzig/Hamburg 1914) XXXV-XL

- «Alfred Hoche †», Deutsche Zeitschrift für Nervenheilkunde 155 (1943) 189-200

- «Wilhelm Erb», in: Kurt Kolle, Grosse Nervenärzte, Bd. 1 (Stuttgart ${ }^{21970) ~ 68-80}$

- Anfang und Ziel meines Lebens. Erinnerungen (Hamburg 1971)

Paetz, Albrecht, Die Kolonisirung der Geisteskranken in Verbindung mit dem Offen-ThürSystem: ihre historische Entwicklung und die Art ihrer Ausführung auf Rittergut Alt-Scherbitz (Berlin 1893)

Panse, Friedrich, «Nekrolog Bratz», Allgemeine Zeitschrift für Psychiatrie 102 (1934) 370-374

Pieper, Christine, Die Sozialstruktur der Chefärzte des Allgemeinen Krankenhauses HamburgBarmbek 1913 bis 1945. Ein Beitrag zur kollektivbiographischen Forschung (Münster/Hamburg/London 2003)

Pistor, Moritz/Sigmund Merkel, «Julius Reincke $\dagger »$, Deutsche Vierteljahresschrift für öffentliche Gesundheitspflege 39 (1906) I-III

Pohlmann, Paul, «Geheimer Sanitätsrat Dr. Backenköhler †», Psychiatrisch neurologische Wochenschrift 26 (1924) 7

Reincke, Johann Julius (Hrsg.), Bericht des Medicinalrathes über die medicinische Statistik des Hamburgischen Staates für das Jahr 1896 (Hamburg 1897)

Rodegra, Heinrich, Das Gesundheitswesen der Stadt Hamburg im 19. Jahrhundert: unter Berücksichtigung der Medizinalgesetzgebung (Wiesbaden 1979)

Rönn, Peter von/Regina Marien-Lunderup/Michael Wunder/Eveline Sonn/Renate Otto/Marc Billhardt/Georg Dahmen, Wege in den Tod. Hamburgs Anstalt Langenhorn und die Euthanasie in der Zeit des Nationalsozialismus, hrsg. von Klaus Böhme und Uwe Lohalm (Hamburg 1993)

Sammet, Kai, «Theodor Neuberger (1856-1938)», in: Franklin Kopitzsch/Dirk Brietzke (Hrsg.), Hamburgische Biographie, Bd. 3 (Hamburg 2005) im Druck

Schmidt, Christian, Die Hamburger Psychiatrie bis 1930 (Diss. med. Würzburg 1974)

Schmiedebach, Heinz-Peter, «Eine <antipsychiatrische Bewegung〉 um die Jahrhundertwende», in: Martin Dinges (Hrsg.), Medizinkritische Bewegungen im Deutschen Reich (ca. 1870ca. 1933) (Stuttgart 1996) 127-159

Schnitzer, Hubert, «Die Irrenanstalt Friedrichsberg», in: Krankenhauskollegium (Hrsg.), Die Allgemeinen Krankenhäuser und Irrenanstalten der Freien und Hansestadt Hamburg (Hamburg 1901) 144-167

Schwingel, Markus, Pierre Bourdieu zur Einführung (Hamburg ${ }^{4} 2003$ )

Sommer, Robert, «Heinrich Adolf Dannemann 1867-1932», Zeitschrift für psychische Hygiene 5 (1932) 129-133

Stimpert, Thomas, Alois Alzheimer (1864-1915). Leben und Werk (Diss. med. Marburg 1994)

Wehler, Hans-Ulrich, Deutsche Gesellschaftsgeschichte, Bd. 3 (München 1995)

Weisser, Ursula/Gordon Uhlmann, «Das Neue Allgemeine Krankenhaus auf dem Eppendorfer Feld - Entstehung und Gestaltung (1879-1888)», in: Ursula Weisser (Hrsg.), 100 Jahre Universitäts-Krankenhaus Eppendorf 1889-1989 (Tübingen 1989) 13-34

Weygandt, Wilhelm, «Theodor Kaes $\dagger »$, Psychiatrisch neurologische Wochenschrift 15 (1914) 613-615

- Friedrichsberg. Staatskrankenanstalt und Psychiatrische Universitätsklinik in Hamburg (Hamburg 1922)

- «Zum 70. Geburtstag von Johannes Bresler», Psychiatrisch neurologische Wochenschrift 38 (1936) 49-53

Wollenberg, Robert, Erinnerungen eines alten Psychiaters (Stuttgart 1931)

Wrede, Richard, Das geistige Berlin, Bd. 3: Leben und Wirken der Ärzte, Apotheker, Baumeister, Ingenieure, Militärschriftsteller, Naturwissenschaftler (Berlin 1898)

Wulf, Stefan, «Max Nonne», Neue Deutsche Biographie, Bd. 19 (Berlin 1999) 333-335 